\title{
Orbiting black-hole binaries and apparent horizons in higher dimensions
}

\author{
William G. Cook $^{1}$, Diandian Wang ${ }^{1}$, Ulrich Sperhake ${ }^{1,2,3}$ \\ ${ }^{1}$ Department of Applied Mathematics and Theoretical Physics, Centre for \\ Mathematical Sciences, University of Cambridge, Wilberforce Road, Cambridge \\ CB3 0WA, United Kingdom \\ 2 Theoretical Astrophysics 350-17, California Institute of Technology, $1200 \mathrm{E}$ \\ California Boulevard, Pasadena, CA 91125, USA \\ 3 Department of Physics and Astronomy, University of Mississippi, University, \\ Mississippi 38677, USA \\ E-mail: U.Sperhake@damtp.cam.ac.uk
}

\begin{abstract}
We study gravitational wave emission and the structure and formation of apparent horizons in orbiting black-hole binary systems in higherdimensional general relativity. For this purpose we present an apparent horizon finder for use in higher dimensional numerical simulations and test the finder's accuracy and consistency in single and binary black-hole spacetimes. The blackhole binaries we model in $D=6$ dimensions complete up to about one orbit before merging or scatter off each other without formation of a common horizon. In agreement with the absence of stable circular geodesic orbits around higherdimensional black holes, we do not find binaries completing multiple orbits without finetuning of the initial data. All binaries radiate about $0.13 \%$ to $0.2 \%$ of the total mass-energy in gravitational waves, over an order of magnitude below the radiated energy measured for four-dimensional binaries. The low radiative efficiency is accompanied by relatively slow dynamics of the binaries as expected from the more rapid falloff of the binding gravitational force in higher dimensions.
\end{abstract}




\section{Introduction}

Einstein's theory of general relativity (GR) in four spacetime dimensions is an extraordinarily successful theory of gravity and has passed a wealth of tests from solar system dynamics to the bending of light and the recent detection of gravitational waves (GWs) by LIGO [1, 2, 3, 4, 5]. While all these tests naturally employ the theory of general relativity in $D=4$ spacetime dimensions, there is a priori nothing special about the choice $D=4$. Rather, $D$ appears merely as a free parameter in the theory [6] which furthermore preserves its fundamental mathematical properties - well posedness, diffeomorphism invariance etc. - for any value of $D$. In recent decades, higher-dimensional GR with $D>4$ has indeed attracted a lot of attention in many areas of physics research.

Much of the interest in theories with extra dimensions is motivated by the quest for a unified theory encompassing all physical interactions and dates back to the pioneering studies of Kaluza and Klein in the 1920s [7, 8]. Extra dimensions naturally appear in String/M theory $[9,10]$ and to some extent in Loop Quantum Gravity [11]. A particularly fruitful concept arising from these developments is the holographic principle according to which the information of a $D$ dimensional gravitational system is encoded in its $D-1$ dimensional boundary. In the form of the Anti-de Sitter/Conformal Field Theory (AdS/CFT) correspondence [12] and similar dualities, holography has inspired a great deal of numerical and analytic explorations of higher dimensional spacetimes containing black holes (BHs) to gain insight into phenomena such as confinement phase transitions in gauge theories, non-equilibrium dynamics of quark-gluon plasma or superconductors; see e.g. [13, 14, 15]. Extra dimensions also play a critical role in attempts to explain the hierarchy problem of physics, i.e. the extraordinary weakness of gravity compared to the other fundamental interactions of physics. In these scenarios, gravity is diluted at sub-millimetre scales due to the presence of extra dimensions such that the fundamental Planck scale is lowered from its effective four-dimensional value of about $10^{19} \mathrm{GeV}$ to the order of Tera electron volts $(\mathrm{TeV})[16,17,18,19,20]$. In these so-called $\mathrm{TeV}$ gravity scenarios, gravity would become the dominant interaction at energies accessible in particle collisions at the Large Hadron Collider (LHC) or the interaction of cosmic rays with the Earth's atmosphere. Despite constraints obtained at current LHC energies $[21,22]$, this leaves open the possibility of $\mathrm{BH}$ production in controlled experiments and, thus, a direct search for evidence of extra dimensions [23, 24, 25]. A quantitative understanding of the dynamics of $\mathrm{BH}$ collisions in higher $D$, particularly the generation of gravitational waves and the formation of a common $\mathrm{BH}$ horizon, is essential in the interpretation of experimental data [26] and forms one of the main motivations for our work.

Higher dimensional GR has also motivated a large amount of mathematical studies and revealed important insight into the theory. The space of solutions to the Einstein equations is exceptionally rich in $D>4$ compared to the standard fourdimensional case, including topologically non-spherical $\mathrm{BH}$ solutions such as black rings or black Saturns [27, 28], but also results in a wider class of instabilities (including generic violation of cosmic censorship) limiting the viability of these solutions [29, 30, 31, 32, 33]. In a complementary approach, Emparan et al. have used the dimensionality $D$ as an expansion parameter focusing in particular on the large- $D$ limit in the resulting series expansion in $1 / D[6,34,35,36]$. Aside from probing gravity in generic dimensions, their approach also recovers rather accurately 
the physics of GR in $D=4$. Further details on all these developments in higherdimensional GR can be found in the review articles [37, 38, 39, 40, 26, 41].

A key goal for numerical relativity in any given setting, such as higher dimensions, is to have a full set of diagnostic tools for that setting. One such tool, and a main focus of this work, is the ability to find the horizon of a BH. Once we can find the horizon, we can then find a measure of the BH's spin and mass, independent of, for instance, wave extraction calculations. The event horizon of a $\mathrm{BH}$ is a gauge independent object, but one that requires knowledge of future null infinity to compute; see [42, 43]. From a practical point of view, this involves considerable challenges in numerical time evolutions, and most applications instead resort to the computation of apparent horizons ( $\mathrm{AH})$; we follow this approach here. The $\mathrm{AH}$ is a slicing dependent object, that always lies on or within the event horizon of the $\mathrm{BH}$, defined as the outermost marginally trapped surface in the spacetime. Developing efficient, accurate horizon finding has been an important area of research within numerical relativity, especially in four spacetime dimensions, with key references including [44, 45, 46, 47, 48]; for a review see [49]. Here we follow the work of Alcubierre et al. [44], and adapt the algorithm used for $4 \mathrm{D}$ horizon finding to higher dimensions. AH results have been reported in some higher-dimensional numerical relativity computations $[30,50,32,33,33]$, but the only detailed description we are aware of is given in the thesis [51] with a focus on topologically ringlike AHs. In this paper we therefore describe in more detail the algorithm used to find AHs in our simulations of colliding $\mathrm{BH}$ binaries in higher dimensional GR. We apply the AH finder to several types of stationary $\mathrm{BH}$ data, and time evolutions of $\mathrm{BH}$ binaries and estimate the numerical accuracy of the physical results extracted from the horizon. The construction of initial data describing a $\mathrm{BH}$ binary with non-zero orbital angular momentum is a second main goal of this paper and enables us to compute numerically the $\mathrm{AH}$ of a rotating $\mathrm{BH}$ dynamically formed in higher dimensions in a binary coalescence. We conclude our study with a first exploration of inspiraling BH binary configurations in $D=6$ and contrast its phenomenology with that of four-dimensional inspirals as observationally confirmed through GW150914 and other events detected by LIGO/Virgo.

This paper is organised as follows. In Sec. 2, we set the ground by introducing our notation and, in particular, the index ranges required in the dimensional reduction in $D>4$ numerical relativity. Section 3 contains the theoretical framework of the AH computations in $D>4$, the particulars encountered in the dimensional reduction and derives coordinate invariant expressions for extracting the mass and spin in the limit of stationary BHs. In Sec. 4 we test the consistency and accuracy of the AH finder by computing horizons of stationary single $\mathrm{BH}$ spacetimes. As a further test, we calculate the convergence properties of the horizon diagnostics in the merger of orbiting binaries in Sec. 5. There, we also study the dynamics of binaries with different initial values of the angular momentum, compute the gravitational wave energy emitted in the evolution and determine the mass and spin of the merger remnant BHs. We conclude with a summary of our findings and an outlook for future work in Sec. 6. Details of the construction of Bowen-York type initial data for BH binaries with non-zero orbital angular momentum are presented in Appendix A.

\section{Notation}

Let $\left(\mathcal{M}, g_{A B}\right), A, B, \ldots=0, \ldots, D-1$, be a $D$ dimensional spacetime, with a Lorentzian metric that solves the $D$-dimensional Einstein equations in vacuum, with 
vanishing cosmological constant,

$$
G_{A B}=R_{A B}-\frac{1}{2} R g_{A B}=0 .
$$

Here we use units where the gravitational constant and the speed of light $G=c=1$. Following the standard spacetime decomposition of Arnowitt, Deser and Misner $(\mathrm{ADM})$ [52], in the formulation of York [53], we can write the line element as

$$
d s^{2}=g_{A B} d x^{A} d x^{B}=\left(-\alpha^{2}+\beta_{I} \beta^{I}\right) d t^{2}+2 \beta_{I} d x^{I} d t+\gamma_{I J} d x^{I} d x^{J},
$$

where $I, J, \ldots=1, \ldots, D-1$ and $\alpha$ and $\beta^{I}$ denote the lapse function and shift vector respectively, and $\gamma_{I J}$ is the induced spatial metric on hypersurfaces given by $t=$ const. For this choice of coordinates and variables, the Einstein equations (1) result in one Hamiltonian and $D-1$ momentum constraints as well as $D(D-1) / 2$ evolution equations cast into first-order-in-time form by introducing the extrinsic curvature $K_{I J}$ through

$$
\partial_{t} \gamma_{I J}=\beta^{M} \partial_{M} \gamma_{I J}+\gamma_{M J} \partial_{I} \beta^{M}+\gamma_{I M} \partial_{J} \beta^{M}-2 \alpha K_{I J}
$$

For a detailed review of this decomposition see [54, 26].

Fully simulating $D$-dimensional spacetimes is a problem that scales exponentially with the parameter $D$, so we employ a method of dimensional reduction in order to alleviate this computational cost. By considering a restricted class of spacetimes with $S O(D-d)$ rotational symmetry we can use the modified cartoon method $[55,56]$, to reduce our $(D-1)$-dimensional spacelike hypersurface to an effective $d$-dimensional computational domain assuming $S O(D-d)$ isometry. In this case, the extra dimensions and their impact on the dynamics is encoded in a few extra variables representing tensor components in the off-domain directions. Specifically, for the symmetries under consideration here, we can write the spatial components of a rank-2 tensor $T_{I J}$ as

$$
T_{I J}=\left(\begin{array}{cc}
T_{i j} & 0 \\
0 & T_{w w} \delta_{a b}
\end{array}\right),
$$

where $i, j, \ldots=1, \ldots, d$, and $a, b, \ldots=d+1, \ldots, D-1$ and $T_{w w}$ denotes one function whose values we only need to know on the $d$ dimensional computational domain. Tensor components with upstairs indices are decomposed in the same way and one straightforwardly shows that $T^{w w}=\gamma^{w w} \gamma^{w w} T_{w w}$, while the components of vectors in the extra dimensions vanish due to the symmetry: $V^{I}=\left(V^{i}, 0\right)$.

In the calculations and simulations presented in this paper we set $d=3$, restricting our attention to a class of spacetimes encompassing many of the physical phenomena we wish to apply our $\mathrm{AH}$ finder to, such as $\mathrm{BH}-\mathrm{BH}$ inspirals, high energy $\mathrm{BH}$ collisions and BHs spinning in a single plane. We implement the evolution equations in the form given by Baumgarte, Shapiro, Shibata and Nakamura (BSSN) [57] [58], with the particular implementation of the BSSN evolution equations in the modified cartoon used in our code given in [59]. Henceforth, we follow the notation in that work which we summarise as follows.

- Upper case early Latin indices $A, B, C, \ldots$ range over the full spacetime from 0 to $D-1$.

- Upper case middle Latin indices $I, J, K, \ldots$ denote all spatial indices, inside and outside the effective three dimensional computational domain, running from 1 to D -1 . 
- Lower case middle Latin indices $i, j, k, \ldots$ denote indices in the spatial computational domain and run from 1 to 3, i.e. $x^{i}=(x, y, z)$.

- Lower case early Latin indices $a, b, c, \ldots$ denote spatial indices outside the computational domain, ranging from 4 to $D-1$, i.e. $x^{a}=\left(w^{4}, \ldots, w^{D-1}\right)$.

- Greek indices $\alpha, \beta, \ldots$ denote all angular directions and range from 2 to $D-1$.

- In our simulations we refer to two coordinate systems, a Cartesian system, $X^{A}=\left(t, x^{i}, x^{a}\right)$, and a spherical system $Y^{A}=\left(t, r, \phi^{\alpha}\right)$.

- $\nabla_{A}$ denotes the covariant derivative in the full $D$ dimensional spacetime, whereas $D_{I}$ denotes the covariant derivative on a spatial hypersurface and is calculated from the spatial metric $\gamma_{I J}$.

\section{Horizon Finding algorithm}

\subsection{Horizon finding in higher dimensions}

The apparent horizon of a $\mathrm{BH}$ is defined as the outermost marginally trapped surface in the spacetime. Equivalently this is the surface on which the expansion of outgoing normal null geodesics is equal to 0 . In order to find the $\mathrm{AH}$ on a spacelike hypersurface $\Sigma$ we calculate the expansion $\Theta=\nabla_{A} k^{A}$ of a congruence of null geodesics with tangent vector $k^{A}$ moving in the outward normal direction to a surface $S$, with outward unit normal vector $s^{A}$. The calculation of the expansion of this congruence in higher dimensions proceeds identically to the calculation in $4 \mathrm{D}$, and we present it here for completeness, following the derivation of Gundlach [45]. Consider a $D$-dimensional spacetime $\left(\mathcal{M}, g_{A B}\right)$, with covariant derivative $\nabla_{A}$. We foliate this spacetime with $D-1$ dimensional spacelike hypersurfaces $\Sigma_{t}$ with timelike normal $n^{A}$. The induced metric on these hypersurfaces is given by

$$
\gamma_{A B}=g_{A B}+n_{A} n_{B}
$$

with extrinsic curvature

$$
K_{A B}=-\gamma_{A}^{C} \nabla_{C} n_{B}=-D_{A} n_{B},
$$

where $D_{A}$ is the covariant derivative associated to $\gamma_{A B}$. Now let $S$ be a closed, $D-2$ dimensional, spacelike hypersurface of $\Sigma_{t}$, with unit outward spacelike normal $s^{A}$, which is also normal to $n^{A} \cdot \gamma_{A B}$ induces a metric $q_{A B}$ on $S$,

$$
q_{A B}=\gamma_{A B}-s_{A} s_{B} .
$$

Now let us consider the future pointing, null geodesic congruence, whose projection onto $\Sigma_{t}$ is orthogonal to $S$ and $k^{A} . k^{A}$ satisfies the following equations:

$$
k^{A} \nabla_{A} k^{B}=0, \quad k^{A} k_{A}=0,\left.\quad q_{A B} k^{A}\right|_{S}=0 .
$$

In consequence of these conditions, we find that, up to a constant factor here set to 1 without loss of generality,

$$
\left.k^{A}\right|_{S}=s^{A}+n^{A} .
$$

Now we can express the expansion $\Theta$ in terms of $(D-1)+1$ quantities,

$$
\begin{aligned}
\Theta & =g^{A B} \nabla_{A} k_{B}=\left(\gamma^{A B}-n^{A} n^{B}\right) \nabla_{A} k_{B} \\
& =\gamma^{A B} \nabla_{A}\left(s_{B}+n_{B}\right)-\left(k^{A}-s^{A}\right)\left(k^{B}-s^{B}\right) \nabla_{A} k_{B} \\
& =\gamma^{A B} \nabla_{A} s_{B}+\gamma^{A B} \nabla_{A} n_{B}-s^{A} s^{B} \nabla_{A} n_{B} .
\end{aligned}
$$


In a coordinate basis adapted to the space-time split, we can write this equation in terms of spatial components,

$$
\Theta=D_{I} s^{I}+s^{I} s^{J} K_{I J}-K .
$$

The outermost surface upon which $\Theta=0$ everywhere will be our AH. It will prove convenient to parametrize this surface with a function $F\left(x^{I}\right)$, such that our surface is given by the solution to the equation $F\left(x^{I}\right)=0$ and we can write,

$$
s^{I}=\frac{D^{I} F}{|D F|}, \quad|D F|:=\sqrt{D_{J} F D^{J} F},
$$

and Eq. (11) can be reframed as a partial differential equation to be solved for the scalar $F$.

In order to evaluate Eq. (11) in the modified cartoon formalism, we must distinguish between directions inside and those pointing off the 3D computational domain. We can then use the rotational symmetry in the extra dimensions to simplify tensors as described in Sec. 2, and furthermore rewrite derivatives in the extra dimensions in terms of derivatives in our computational domain; the details for this procedure are given in [59]. The only terms in Eq. (11) that will require such treatment of extra dimensional components are $D_{I} s^{I}$ and the trace of the extrinsic curvature. The latter is directly obtained as $K=K^{I}{ }_{I}=K^{i}{ }_{i}+(D-4) \gamma^{w w} K_{w w}$ while we write the former as

$$
\begin{aligned}
D_{I} s^{I} & =D_{i} s^{i}+D_{a} s^{a} \\
& =\frac{D_{i} D^{i} F}{|D F|}-\frac{\left(D^{i} F\right)\left(D_{j} F\right) D_{i} D^{j} F}{|D F|^{3}}+(D-4) \frac{\partial^{z} F}{|D F| z}+\frac{D-4}{2} \gamma^{w w} \partial_{k} \gamma_{w w} \frac{\partial^{k} F}{|D F|} .
\end{aligned}
$$

In summary the equation we will use to solve for $F$ is

$$
\begin{aligned}
0=\Theta= & \frac{D_{i} D^{i} F}{|D F|}-\frac{\left(D^{i} F\right)\left(D_{j} F\right) D_{i} D^{j} F}{|D F|^{3}}+(D-4) \frac{\partial^{z} F}{|D F| z} \\
& +\frac{1}{2}(D-4) \gamma^{w w} \partial_{k} \gamma_{w w} \frac{\partial^{k} F}{|D F|}+\frac{K_{i j} \partial^{i} F \partial^{j} F}{|D F|^{2}}-K .
\end{aligned}
$$

At $z=0$ the term $\partial^{z} F / z$ appears ill-defined. According to the regularisation procedures laid out in Appendix B of [59], however, we can substitute in the limit of small $z$

$$
\lim _{z \rightarrow 0} \frac{\partial^{z} F}{z}=\lim _{z \rightarrow 0} \partial_{z} \partial^{z} F
$$

\subsection{Minimisation Algorithm}

In order to numerically solve Eq. (14), we have extended the minimisation algorithm provided inside the CACTUS Computational Toolkit [60, 61] and described in [44, 62] to the case of $D$ dimensions with $S O(D-3)$ isometry. The first step consists in reparametrizing the function $F$, restricted to the $3 \mathrm{D}$ computational domain, as

$$
F\left(r, \phi^{2}, \phi^{3}\right)=r-h\left(\phi^{2}, \phi^{3}\right) .
$$

We can then expand $h$ in terms of real spherical harmonics $Y_{l m}\left(\phi^{2}, \phi^{3}\right)$.

$$
h\left(\phi^{2}, \phi^{3}\right)=\sum_{l} \sum_{m} \sqrt{4 \pi} a_{l m} Y_{l m}\left(\phi^{2}, \phi^{3}\right) .
$$


The iterative search for a solution starts with a spherical trial function for $h$, from which we calculate $F$, and so $\Theta$, by Eqs. (16), (14). Next, $\Theta$ is interpolated onto the points at which $r=h\left(\phi^{2}, \phi^{3}\right)$, and used to calculate the surface integral of $\Theta^{2}$ over this 2D surface. Powell's minimisation algorithm [63] then leads to the values $a_{l m}$ for which this integral is minimised. Once a function $F$ giving a minimum for $\Theta^{2}$ is found, we must determine whether this is a local or global minimum. Following [44], this is achieved by recalculating the candidate function $F$ with higher spatial resolution, and more terms in the spherical harmonic expansion (17). If the value of the integral of $\Theta^{2}$ continues to decrease to zero, rather than reaching some non-zero limiting value, it is interpreted as a global minimum and the corresponding $F$ defines the $\mathrm{AH}$. The horizon surface then allows us to calculate further diagnostic quantities as described in the next section.

\subsection{Black hole diagnostics}

Once we have found the $\mathrm{AH}$ we wish to extract physical diagnostics of BHs from them. When we consider stationary BHs, such as those produced by exact initial data (e.g. Secs. 4.1 and 4.2), we know that the world tube of the AH coincides exactly with the event horizon, see [64] for the proof in $D=4$, and [65] for a discussion of the generalisation of this, and related proofs, to higher dimensions. For BHs produced as the result of mergers in our simulations (e.g. Sec. 5.1) we assume that the spacetime will, after a long enough period of time, be perturbatively close to a stationary $\mathrm{BH}$, and that in this case the AH will closely approximate the spatial cross section of an event horizon. We therefore base our calculation of $\mathrm{BH}$ mass and spin on the assumption that the spacetime describes a stationary $\mathrm{BH}$.

3.3.1. Non-spinning Black Holes For illustration, we first consider non-rotating BHs in $D$ spacetime dimensions. These are described by the Tangherlini metric [66] given in Schwarzschild coordinates by

$$
d s^{2}=-\left(1-\frac{\mu}{\tilde{r}^{D-3}}\right) d t^{2}+\left(1-\frac{\mu}{\tilde{r}^{D-3}}\right)^{-1} d \tilde{r}+\tilde{r}^{2} d \Omega_{D-2}^{2},
$$

where

$$
\mu=\frac{16 \pi M}{(D-2) \Omega_{D-2}},
$$

is the mass parameter. $d \Omega_{n}$ is the line element on the unit $n$-sphere, parameterised by $n$ angular coordinates, $\left(\phi^{2}, \ldots \phi^{n+1}\right), \Omega_{n}$ is the surface area of the unit $n$-sphere, and $M$ is the ADM mass associated to the spacetime containing the $\mathrm{BH}$ with mass parameter $\mu$. By considering Eq. (18) we can see that the event horizon of the $\mathrm{BH}$ is given by the surface $\tilde{r}_{S}^{D-3}=\mu$. We find the area of this surface to be

$$
A_{\mathrm{hor}}=\int_{\mathcal{H}} \sqrt{q} d \phi^{2} \ldots d \phi^{D-1}=\tilde{r}_{S}^{D-2} \Omega_{D-2},
$$

where $q=\operatorname{det} q_{I J}$, and $A_{h o r}$ is the area of the AH, as this is a stationary BH. Combining this expression with Eq. (19), we find

$$
M=\frac{D-2}{16 \pi} \Omega_{D-2}^{1 /(D-2)} A_{\mathrm{hor}}^{(D-3) /(D-2)} .
$$


3.3.2. Spinning black holes The Myers-Perry metric for a singly spinning BH (the higher-dimensional analogue of the Kerr $\mathrm{BH}$ ) is given by [67]

$$
\begin{aligned}
d s^{2}= & -d t^{2}+\frac{\mu}{r^{D-5} \Sigma}\left(d t-a \sin ^{2} \theta d \tilde{\phi}\right)^{2}+\frac{\Sigma}{\Delta} d r^{2}+\Sigma d \theta^{2} \\
& +\left(r^{2}+a^{2}\right) \sin ^{2} \theta d \tilde{\phi}^{2}+r^{2} \cos ^{2} \theta d \Omega_{D-4}^{2}, \\
\Sigma= & r^{2}+a^{2} \cos ^{2} \theta, \\
\Delta= & r^{2}+a^{2}-\frac{\mu}{r^{D-5}},
\end{aligned}
$$

where $\mu$ is the mass parameter, and $a$ is the spin parameter. Unlike in $4 D$, where the Kerr $\mathrm{BH}$ is the unique uncharged rotating $\mathrm{BH}$ solution, in higher dimensions other solutions with the same mass and spin, such as black rings [27], or black Saturns [28] can exist. In the discussion of binary mergers below, we assume that the end product is a Myers-Perry BH. As we shall see, this expectation is borne out by the results of the AH finder. In our notation, the angular coordinates of Eq. (22) are $\phi^{2}=\theta, \phi^{3}=\tilde{\phi}$ and $\phi^{4}, \ldots \phi^{D-1}$ denote the angular coordinates on the $(D-4)$-sphere in the metric. The ranges of the angular coordinates are $\theta \in[0, \pi / 2], \tilde{\phi}, \phi^{D-1} \in[0,2 \pi]$ and all other angles lie in the interval $[0, \pi]$. The location of the horizon is given by the largest root of $\Delta=0$

$$
\frac{\mu}{r_{+}^{D-5}}=r_{+}^{2}+a^{2}
$$

and, following a brief calculation, the horizon area is, similarly to Eq. (20), given by

$$
A_{\text {hor }}=\int_{\mathcal{H}} \sqrt{q} d \theta d \tilde{\phi} d \phi^{4} \ldots d \phi^{D-1}=r_{+}^{D-4}\left(r_{+}^{2}+a^{2}\right) \Omega_{D-2}=r_{+} \mu \Omega_{D-2} .
$$

To calculate the spin we will need the equatorial circumference

$$
\ell_{e}=\int_{0}^{2 \pi} \sqrt{g_{\tilde{\phi} \tilde{\phi}}} d \tilde{\phi}=2 \pi \frac{r_{+}^{2}+a^{2}}{r_{+}}=2 \pi \frac{\mu}{r_{+}^{D-4}},
$$

giving us

$$
r_{+}^{D-3}=\frac{2 \pi}{\Omega_{D-2}} \frac{A_{\text {hor }}}{\ell_{e}}, \quad \mu=\frac{A_{\text {hor }}}{r_{+} \Omega_{D-2}}, \quad a=\sqrt{\frac{\mu}{r_{+}^{D-5}}-r_{+}^{2}} .
$$

Note that Eq. (19) holds also for stationary, spinning BHs. Substituting $\mu$ in that expression in terms of $\Omega_{D-2}$ and $A_{\text {hor }}$ and finally setting $\ell_{e}=2 \pi r_{S}, r_{+}=r_{S}$ for the non-spinning limit, one indeed recovers Eq. (21). In order to obtain a dimensionless quantity for the BH rotation, we follow Eqs. (21), (22) in [37] and define the spin parameter

$$
j=c_{J}^{1 /(D-3)} \frac{J}{M^{1 /(D-3)} M}, \quad c_{J}=\frac{\Omega_{D-3}}{2^{D+1}} \frac{(D-2)^{D-2}}{(D-3)^{(D-3) / 2}}, \quad J=\frac{2}{D-2} M a .
$$

For $D=5$ this implies $j=a / \sqrt{\mu}$ and, hence, $j=1$ for an extremal Myers-Perry BH. $\ddagger$ In $D=4$ spacetime dimensions, however, Eq. (26) yields $j=a \pi /(2 \mu)=a \pi /(4 M)$ and one might instead use the more standard $j \equiv a / M$. Since all BH spacetimes discussed in this work have $D \geq 5$ spacetime dimensions, we employ (26) throughout.

$\ddagger$ For BHs in $D \geq 6$ rotating in a single plane, there exists no upper limit for the spin magnitude and therefore no extremal configuration one might naturally wish to identify with $j=1$. 


\section{Computing horizons in single-black-hole spacetimes}

The numerical simulations discussed in this and the following section have been performed with the LEAN code $[68,69]$ based on the CACTUS computational toolkit [60, 61] and using mesh refinement provided by CARPET [70, 71]. The LEAN code was originally developed for $\mathrm{BH}$ simulations in $D=4$ dimensions, using the moving puncture method [72, 73], and upgraded to general $D$ spatial dimensions with $S O(D-3)$ isometry in $[74,59,75]$. Here we use the modified cartoon implementation originally presented in [55]; see also [56].

The first two tests of the AH finder involve analytic initial data for spacetimes containing a single $\mathrm{BH}$. We test a Schwarzschild-Tangherlini $\mathrm{BH}$ in 5 dimensions with initial data constructed using isotropic coordinates, and a 5 dimensional singly spinning Myers-Perry BH with initial data in Kerr-Schild coordinates. In the first example we use the horizon mass as a diagnostic for the AH finder, and in the second we use the horizon mass and spin to analyse the accuracy of our horizon finder.

\subsection{Isotropic Schwarzschild-Tangherlini}

In Schwarzschild coordinates the Schwarzschild-Tangherlini metric (18) is singular at the event horizon. These coordinates are not suitable for a numerical computation of the horizon and we consequently change to isotropic coordinates (see e.g. [76]),

$$
\tilde{r}=r\left(1+\frac{\mu}{4 r^{D-3}}\right)^{2 /(D-3)},
$$

which results in the line element

$$
d s^{2}=-\left(\frac{4 r^{D-3}-\mu}{4 r^{D-3}+\mu}\right)^{2} d t^{2}+\left(1+\frac{\mu}{4 r^{D-3}}\right)^{4 /(D-3)}\left(d x^{2}+d y^{2}+d z^{2}+\sum_{a} d w_{a}^{2}\right) .
$$

Here $r^{2}=\sum_{I}\left(x^{I}\right)^{2}$ is the isotropic radius and there is now no coordinate singularity at the horizon. We perform the ADM spacetime decomposition, picking the isotropic time coordinate as the time coordinate of our foliation, from which we can read off our initial data,

$$
\begin{array}{ll}
\alpha=\frac{4 r^{D-3}-\mu}{4 r^{D-3}+\mu}, & \beta^{I}=0, \\
\gamma_{I J}=\delta_{I J}\left(1+\frac{\mu}{4 r^{D-3}}\right)^{4 /(D-3)}, & K_{I J}=0 .
\end{array}
$$

We use our horizon finder to calculate the $\mathrm{BH}$ mass for a single isotropic Schwarzschild-Tangherlini $\mathrm{BH}$ for a grid configuration with 2 nested grids with radii $\{(4,2) \times(), h\}$, using the notation of Sec. II $\mathrm{F}$ in [68], in units of the Schwarzschild radius $r_{S}=\mu^{1 /(D-3)}$. Such a small grid would be impractical for time evolutions. Here, however, the grid merely serves as a discretized subset of the hypersurface of constant time from which the horizon finder obtains through second-order interpolation the spacetime metric and extrinsic curvature in its iterative computation of the outermost trapped surface. The uncertainty in the calculation of the $\mathrm{AH}$ is then dominated by the error incurred in the interpolation and the secondorder discretization employed inside the $\mathrm{AH}$ finder.

In order to quantify the accuracy of the $\mathrm{AH}$ finder, we vary the grid resolution $h$ on the inner refinement level and correspondingly increase the number of angular 


\begin{tabular}{|c|ccc|}
\hline$h / r_{S}$ & $1 / 8$ & $1 / 16$ & $1 / 32$ \\
\hline$M_{\mathrm{hor}} / M_{A D M}$ & 1.001380 & 1.000237 & 1.000008 \\
\hline$Q$ & $Q_{2}=4.00$ & $Q=4.99$ & \\
\hline
\end{tabular}

Table 1. Measured horizon mass of a Schwarzschild-Tangherlini BH at different resolutions. The convergence factor $Q$ is computed according to Eq. (31) and shows good agreement with the value $Q_{2}=4$ expected for second-order convergence.

points in the AH finder from 50 to 200. The results are listed in Table 1 and enable us to compute the convergence factor $Q$ given by

$$
Q=\frac{M_{1 / 8}-M_{1 / 16}}{M_{1 / 16}-M_{1 / 32}}
$$

where $M_{h}$ is the value of the horizon mass calculated for a given resolution $h$. Inserting the values of Table 1 gives us $Q=4.99$, close to the value $Q_{2}=4$ expected for the second-order discretization in the $\mathrm{AH}$ finder.

\subsection{D Myers-Perry in Kerr-Schild coordinates}

As in the case of the Schwarzschild $\mathrm{BH}$, the numerical calculation of the $\mathrm{AH}$ of a spinning Myers-Perry BH requires coordinates that are not singular at the $\mathrm{BH}$ horizon. One such set of coordinates are Kerr-Schild coordinates, in which the metric is written in the form

$$
d s^{2}=\left(\eta_{A B}+H l_{A} l_{B}\right) d x^{A} d x^{B},
$$

for appropriate $H$, and null vector $l_{A}$. Let us specifically consider a $D=5$ singly spinning Myers-Perry BH in Cartesian coordinates $(t, x, y, z, w)$. The spin parameter is $a$ and the spin lies purely in the $x-y$ plane. Following [67] we can write this metric in Kerr-Schild form, with the functions in Eq. (32) given by

$$
H=\frac{\mu r^{2}}{\Pi F}, \quad \Pi=r^{2}\left(r^{2}+a^{2}\right), \quad F=1-\frac{a^{2}\left(x^{2}+y^{2}\right)}{\left(r^{2}+a^{2}\right)^{2}},
$$

and

$$
l_{A}=\left(1, \frac{r x+a y}{r^{2}+a^{2}}, \frac{r y-a x}{r^{2}+a^{2}}, \frac{z}{r}, \frac{w}{r}\right),
$$

where $r$ is given by the solution to the equation $l_{A} l^{A}=0$, i.e. $r^{4}-r^{2}\left(\rho^{2}-a^{2}\right)-a^{2}\left(z^{2}+\right.$ $\left.w^{2}\right)=0$, where $\rho^{2}=x^{2}+y^{2}+z^{2}+w^{2}$. Using the same setup as for the SchwarzschildTangherlini $\mathrm{BH}$ we foliate the spacetime with slices of constant Kerr-Schild time, $t$, read off the induced metric from the line element, and calculate the initial extrinsic curvature, which for brevity we do not reproduce here.

In Table 2 we present the calculated angular momentum and mass for the MyersPerry $\mathrm{BH}$ for different resolutions and spin values. The convergence factors listed in the table are calculated in analogy to Eq. (31) above and yield good agreement with second-order convergence, but note that we require overall higher grid resolution to achieve comparable accuracy if the BHs are spinning. 


\begin{tabular}{|c|ccc|ccc|}
\hline$a / \sqrt{\mu}$ & & 0.1 & & & 0.9 & \\
$h / r_{\mathrm{S}}$ & $1 / 16$ & $1 / 32$ & $1 / 64$ & $1 / 32$ & $1 / 48$ & $1 / 64$ \\
\hline$M_{\text {hor }} / M$ & 1.0005025 & 1.0001200 & 1.0000287 & 1.0012295 & 1.0003776 & 1.0000498 \\
$j_{\text {hor }}$ & 0.1007076 & 0.1001868 & 0.1000571 & 0.8979883 & 0.8991569 & 0.8995459 \\
\hline$Q$ & $Q_{2}=4$ & $Q_{M}=4.02$ & $Q_{J}=4.19$ & $Q_{2}=2.86$ & $Q_{M}=2.60$ & $Q_{J}=3.00$ \\
\hline
\end{tabular}

Table 2. Horizon mass $M_{\text {hor }}$ and the extracted dimensionless spin parameter $j_{\text {hor }}=a_{\text {hor }} / \sqrt{\mu}$ as obtained for a Myers-Perry BH in Kerr-Schild coordinates (32) in $D=5$ with $a / \sqrt{\mu}=0.1$ and 0.9. The bottom row lists the expected convergence factor $Q_{2}$ for second-order convergence and the measured convergence factors $Q_{f}=\left(f_{h_{1}}-f_{h_{2}}\right) /\left(f_{h_{2}}-f_{h_{3}}\right)$ for mass $(f=M)$ and spin $(f=J)$. For the large spin $a / \sqrt{\mu}=0.9$, we require higher grid resolution to find the $\mathrm{AH}$; hence the different range of resolutions used in the convergence analysis.

\section{Black-hole binaries}

We now consider the dynamic formation of a rotating $\mathrm{BH}$ through the coalescence of a BH binary with non-vanishing initial orbital angular momentum or, equivalently, with non-zero impact parameter $b$. This study serves two purposes: (i) to test the $\mathrm{AH}$ finder in a dynamic scenario where a rotating $\mathrm{BH}$ forms and gradually settles down into a stationary configuration; (ii) to perform a first exploration of the dynamics of orbiting $\mathrm{BH}$ binary systems in higher dimensions.

Before we quantitatively analyse these configurations, however, we emphasise a few important points about orbiting binaries in $D>4$ dimensions. In general, we expect this type of $\mathrm{BH}$ collisions to yield similar regimes of scattering and merging configurations in $D>4$ as known in $D=4$ [77, 78]: below a scattering threshold, $b<b_{\text {scat }}$, the binary results in a merger while for $b>b_{\text {scat }}$, the constituents will scatter off to infinity. This behaviour has been observed for $D=5$ grazing collisions in Ref. [50]. Even without numerical simulations, however, we immediately notice two major differences between inspirals or grazing collisions in $D=4$ as compared with their $D>4$ counterparts. (i) Unlike in $D=4$, there exist no stable circular orbits around a Myers-Perry $\mathrm{BH}$ in $D>4$ [79], ruling out, for instance, stable planetary orbits in a $D>4$ solar system. Viable gravity theories based on higherdimensional GR therefore require some kind of screening mechanism limiting the impact of extra dimensions to very large or small scales. (ii) The second difference is of quantitative nature and concerns the relatively weaker gravitational binding force in binary systems in $D>4$. For any BH binary whose orbit begins close to an unstable circular orbit, this implies correspondingly weaker centrifugal forces and, thus, that the orbital velocity in the inspiral will be much slower than in $D=4$. This is, of course, a special manifestation of the well-known result that in the large $D$ limit, there is no gravitational force outside the horizon; cf. [6]. In practice, both features manifest themselves in the dependence of the binary dynamics on the initial momentum parameters: (i) we need relatively small initial momenta lest the binary scatters rather than merges and (ii) without careful finetuning of the initial momentum, we find it hard to obtain inspirals completing more than a small fraction of an orbit prior to a rapid plunge phase.

We note that in [50], grazing $\mathrm{BH}$ collisions have been studied in $5 D$. In this work it was noted that no "zoom-whirl" orbits were found in $5 D$. These orbits have been identified in numerical studies in $4 D$, where inspiraling $\mathrm{BHs}$ whirl around each other for a number of orbits before either merging or scattering to infinity [80, 81, 82, 78]. 
Though we cannot make a statement on the existence of such orbits generically in higher dimensions without fully exploring the parameter space of initial momenta and impact parameters, and in particular investigating high energy grazing collisions, we note that the sharp transition between scattering orbits and mergers that only involve a single orbit supports the hypothesis that such zoom-whirl orbits cannot be formed in higher dimensions.

Bearing in mind these considerations, we numerically model orbiting binaries and compute the $\mathrm{AH}$ of post-merger remnant $\mathrm{BHs}$. We first need initial data describing a realistic snapshot of a $\mathrm{BH}$ binary in orbit.

\subsection{Numerically constructed Bowen-York like data for BH inspiral in $D>4$}

In constructing initial data for spacetimes containing multiple BHs with linear or angular momentum, we follow the Bowen-York ansatz [83, 84] commonly employed in $D=4$. Assuming a conformally flat metric, the constraint equations are numerically solved with a particular ansatz to give initial data that approximate boosted or spinning BHs. Following Yoshino et al. [85] and Zilhão et al. [86] we implement initial data for 2 Schwarzschild-Tangherlini BHs (non-spinning) each with linear momentum. Specifically, we extend the formalism of [86] to arbitrary directions of the initial linear momentum vector and, correspondingly, non-zero initial orbital angular momentum. We thus construct inspiraling binaries (rather than head-on collisions), whose coalescence we expect to result in a singly spinning, Myers-Perry BH.

This is achieved by assuming the spatial metric is conformally flat, and the extrinsic curvature is tracefree,

$$
\begin{aligned}
& \gamma_{I J}=\psi^{4 /(D-3)} \delta_{I J}, \\
& K_{I J}=\psi^{-2} \hat{A}_{I J},
\end{aligned}
$$

where $\psi$ is a conformal factor, and $\hat{A}_{I J}$ is the conformally rescaled trace free part of the extrinsic curvature. We then make the ansatz

$$
\begin{aligned}
\hat{A}^{I J}= & \frac{4 \pi(D-1)}{(D-2) \Omega_{D-2}} \frac{1}{r^{D-2}} \\
& {\left[n^{I} P^{J}+n^{J} P^{I}-n_{M} P^{M} \hat{\gamma}^{I J}+(D-3) n^{I} n^{J} P^{M} n_{M}\right], }
\end{aligned}
$$

where $P^{I}$ is the momentum vector of the $\mathrm{BH}$ and $n^{I}$ is the normal radial vector. Finally we solve for $\psi$ in the constraint equation by means of an elliptic PDE solver provided by the Cactus thorn TwoPunctures [87]. For further details on this initial data see Appendix A.

\subsection{Apparent horizons of merged black-hole binary}

Based on this initial data construction, we have evolved in $D=6$ dimensions the set of binary configurations summarised in Table 3. In order to obtain dimensionless numbers, we have normalised the parameters and results for our binary configurations as follows. Mass and energy are expressed in units of the ADM mass $M$. Through Eq. (19) and the relation $r_{\mathrm{S}}=\mu^{1 /(D-3)}$, we obtain the Schwarzschild radius associated with the value of the ADM mass and we express length and time in units of $r_{\mathrm{S}}$. Likewise, we use the single BH relation (26) to associate a global, dimensionless angular momentum parameter $j_{\mathrm{gl}}$ to the spacetime's total angular momentum $J$. Up to a 


\begin{tabular}{|c|ccc|cccc|}
\hline & $x_{0} / r_{\mathrm{S}}$ & $j_{\mathrm{gl}}$ & $h / r_{\mathrm{S}}$ & $M_{\text {hor }} / M$ & $j_{\text {hor }}$ & $E_{\mathrm{rad}} / M$ & $t_{\mathrm{m}} / r_{\mathrm{S}}$ \\
\hline A1 & 3.185 & 0.1646 & $1 / 64$ & 0.9986 & 0.1597 & $1.969 \times 10^{-3}$ & 137 \\
A2 & 3.185 & 0.1646 & $1 / 96$ & 0.9984 & 0.1577 & $1.975 \times 10^{-3}$ & 136 \\
A3 & 3.185 & 0.1646 & $1 / 128$ & 0.9984 & 0.1573 & $1.975 \times 10^{-3}$ & 135 \\
\hline B1 & 6.186 & 0.1271 & $1 / 96$ & 1.0014 & 0.1215 & $1.376 \times 10^{-3}$ & 836 \\
B2 & 6.186 & 0.1362 & $1 / 64$ & 0.9986 & 0.1373 & $1.471 \times 10^{-3}$ & 2158 \\
B3 & 6.186 & 0.1362 & $1 / 96$ & 0.9994 & 0.1356 & $1.549 \times 10^{-3}$ & 1738 \\
B4 & 6.186 & 0.1362 & $1 / 128$ & 0.9997 & 0.1352 & $1.558 \times 10^{-3}$ & 1612 \\
B5 & 6.186 & 0.1408 & $1 / 96$ & - & - & $5.7 \times 10^{-5}$ & - \\
\hline
\end{tabular}

Table 3. Summary of the BH binary configurations simulated in $D=6$ dimensions. We characterise a simulation by the initial $\mathrm{BH}$ location $\pm x_{0}$, the initial angular momentum parameter $j_{\mathrm{gl}}$ and the resolution $h$ on the innermost refinement level. The diagnostic variables are the mass $M_{\text {hor }}$ of the common AH (if one forms), the dimensionless spin parameter $j_{\text {hor }}$ of the post-merger $\mathrm{BH}$, the energy $E_{\text {rad }}$ radiated in gravitational waves and an estimate $t_{\mathrm{m}}$ for the time to formation of a common horizon obtained here as the retarded time corresponding to the maximum in the energy flux $d E_{\mathrm{rad}} / d t$. This estimate for $t_{\mathrm{m}}$ agrees within a few $r_{\mathrm{S}}$ with the first time the $\mathrm{AH}$ finder reports a common $\mathrm{AH}$.

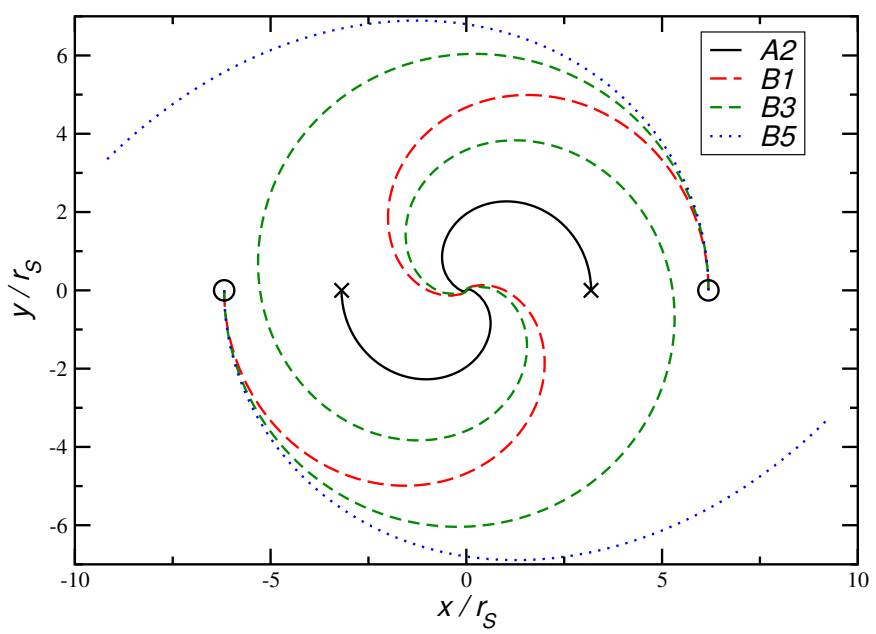

Figure 1. Puncture trajectories of simulations A2, B1, B3 and B5 from Table 3. The crosses (circles) mark the initial $\mathrm{BH}$ positions of configurations A2 (B1, B3, B5).

geometric factor of order unity, $j_{\mathrm{gl}}$ measures the total angular momentum per ADM mass raised to the power of $(D-2) /(D-3)$. In summary, we have

$$
r_{\mathrm{S}}^{D-3} \equiv \frac{16 \pi M}{(D-2) \Omega_{D-2}}, \quad j_{\mathrm{gl}} \equiv c_{J}^{1 /(D-3)} \frac{J}{M^{(D-2) /(D-3)}} .
$$

We first consider the binaries labelled A1 to A3 in Table 3 where two non-spinning, equal-mass BHs start positions $x_{0} / r_{S}= \pm 3.185$ with opposite linear momentum in the $y$ direction corresponding to an angular momentum $j_{\mathrm{gl}}=0.1646$. The grid setup is $\left\{(160,120,72,24,12,6) \times(1.25,0.625), h_{i}\right\}$ and the three simulations differ in the grid resolution: $h_{1}=r_{S} / 64, h_{2}=r_{S} / 96$ and $h_{3}=r_{S} / 128$. The trajectory traced out by the binary configuration A2 is shown as the solid, black curve in Fig. 1. 

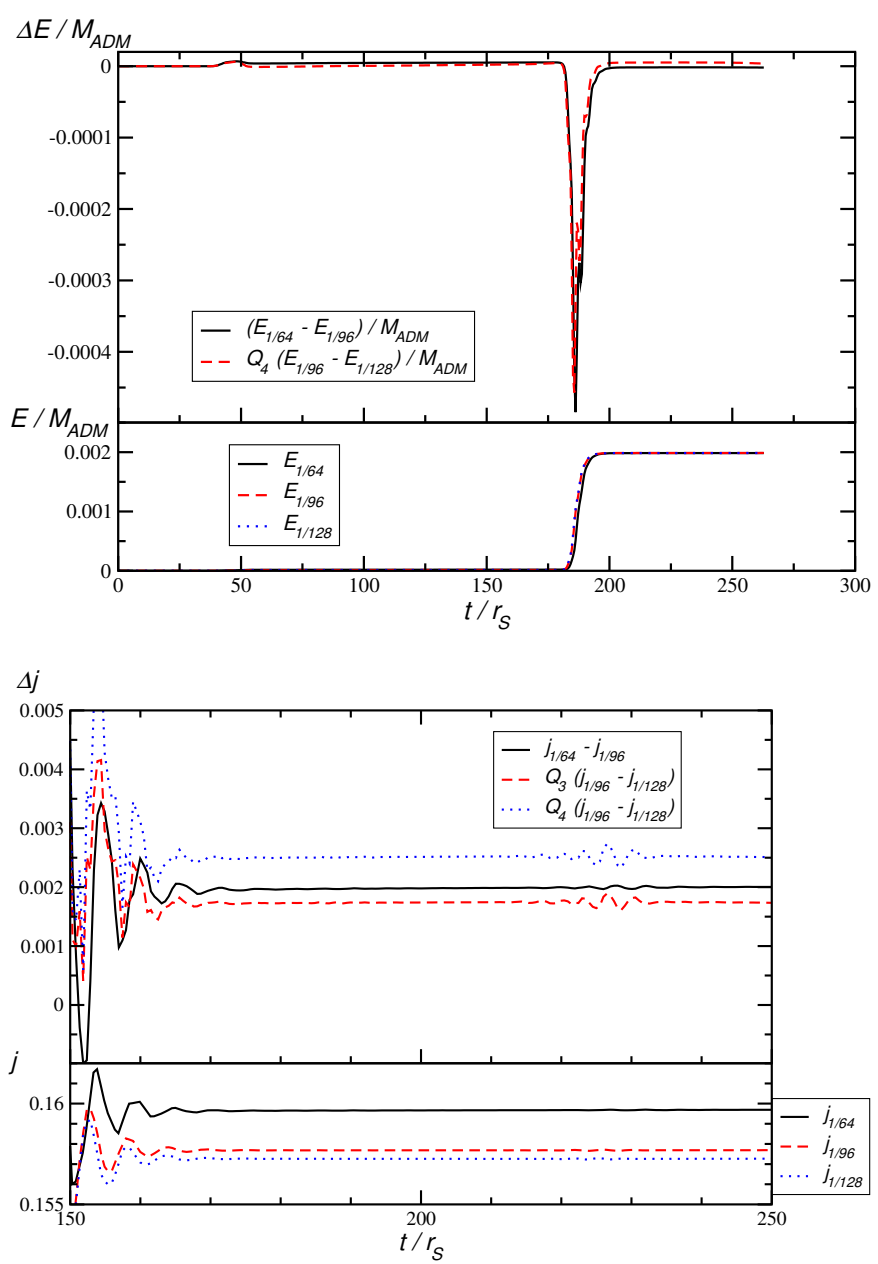

Figure 2. Analysis of the energy radiated in gravitational waves (top) and the merger remnant's dimensionless spin (bottom). For either quantity, we show results obtained for the three grid resolutions in the lower panel and compare in the upper panel the differences low-medium vs. medium-high resolution, rescaling the latter by a factor $Q_{4}=5.94$ or $Q_{3}=4.11$ expected for 4 th or 3rd order convergence.

Following the procedure described in [75], we have calculated the energy emitted in gravitational waves in these simulations and for the post-merger phase (starting at about $t / r_{S}=150$ ), we extract the spin of the merger remnant as detailed in Sec. 3.3.2 above. The results obtained for the different resolutions are shown in Fig. 2 together with an analysis of the respective convergence properties. We find the radiated energy to converge at 4th order and the spin between 3rd and 4th order which is in agreement with the 4th order discretization used in the time evolution and indicates that the error budget here is dominated by the uncertainty of the $\mathrm{BH}$ evolution rather than the $\mathrm{AH}$ finder itself. This is confirmed by the relatively larger uncertainties of the mass and spin measurements as compared with those obtained for the single BH cases in Tables 1 and 2. Comparison with the corresponding Richardson extrapolated values 
gives us an uncertainty estimate of $1.8 \%(0.5 \%, 0.22 \%)$ for the dimensionless spin at low (medium, high) resolution. We likewise obtain a peak discretization error of $5 \%(2 \%)$ for the radiated energy $E_{\text {rad }}$ computed for medium (high) resolution, but note that this error is largely due to the small differences in the time to merger and the sudden jump of $E_{\mathrm{rad}}$ around that time; the uncertainty in the total radiated energy is smaller, $1 \%$ or less for all resolutions used here. A further source of uncertainty in the radiated energy is due to finite extraction radii and determined here as about $2 \%$ following Ref. [88]. Combining these uncertainties, we obtain for configuration A3 $E_{\mathrm{rad}} / M_{\mathrm{ADM}}=0.199 \pm 0.005 \%$, about twice as much as the head-on value $E_{\mathrm{rad}, \mathrm{ho}} / M_{\mathrm{ADM}}=0.0819 \%[89,75]$.

\subsection{Orbiting BH binaries}

The BH binary discussed in the previous subsection completed less than half an orbit before coalescing into a single $\mathrm{BH}$. This may in part be due to the relatively small initial separation and we now consider binaries starting from larger initial distance given by $\pm x_{0} / r_{\mathrm{S}}=6.186$. These models are labelled B1-B5 in Table 3, start with angular momentum parameters $j_{\mathrm{gl}}=0.1271,0.1362$ and 0.1408 , respectively, and have been evolved with a grid setup $\left\{(640,320,160,120,72,24,12) \times(2.5,1.25,0.625), h_{i}\right\}$. The table lists the mass and spin parameters $M_{\text {hor }}, j_{\text {hor }}$ obtained from the common $\mathrm{AH}$ formed in the coalescence as well as the radiated energy $E_{\mathrm{rad}}$. We also estimate the time it takes the binaries to merge by locating the peak value of the radiated energy flux $d E_{\mathrm{rad}} / d t$ in retarded time. We find these values to be in excellent agreement a few $r_{S}$ - with the times the AH finder first finds a common AH. For configuration B5, we do not find a common horizon which is corroborated by the binary trajectories shown in Fig. 1. Although we cannot strictly rule out that binary B5 might merge at a much later time, the blue, short-dashed trajectories corresponding to $j_{\mathrm{gl}}=0.1408$ indicate a scattering rather than a merging binary. In contrast, binary B1 shows a behaviour similar to that of A2; the two BHs complete barely more than half an orbit before merging into a single $\mathrm{BH}$. Increasing the initial angular momentum to the value $j_{\mathrm{gl}}=0.1362$ of case B3, leads to a longer inspiral phase (cf. $t_{\mathrm{m}}$ in Table 3 ), but we still find the binary to merge after only $\sim 1$ orbit.

These findings are compatible with the unstable character of timelike circular geodesics around $\mathrm{BHs}$ in higher dimensions. By finetuning the angular momentum parameter, it may be possible to obtain binary systems orbiting multiple times before merger, but small deviations from such a finetuned value appear to result either in a rapid plunge (case B3) or a fly-by (case B5). We note in this context that the time-to-merger $t_{\mathrm{m}}$ depends rather sensitively on the numerical resolution employed (cf. cases B2 and B4) while the numerical errors in the other diagnostic quantities remain small. Close to the threshold that separates mergers from scattering binaries, even small variations in the angular momentum (such as those arising from numerical error) can lead to considerable differences in the trajectories. A more comprehensive understanding of higher dimensional binaries around the threshold value of $j_{\mathrm{gl}}$ clearly requires a larger number of simulations and we propose that the numerical convergence near the threshold then be tested as in Ref. [78] through sequences of binaries rather than one individual configuration.

We finally note the drastically different character of the dynamics we observe here in $D=6$ dimensions as compared with four spacetime dimensions. In $D=4 \mathrm{BH}$ spacetimes have stable circular orbits and variations in the angular momentum lead 
to a continuous transition from inspiraling to plunging binaries that complete many orbits (depending on initial separation); see e.g. Ref. [69]. The attractive character of quasi-circular orbits has long since been known in $D=4$ general relativity as binaries are more efficient in radiating angular momentum than energy [90]. The $D=6$ binaries we have studied here, in contrast, appear to rapidly scatter off each other or plunge towards merger instead of approaching a quasi-circular orbit.

As a further consequence of the stronger falloff of gravity in $D>4$, we note the substantially weaker amount of gravitational radiation emitted in the $D=6$ binaries studied here: our merging configurations radiate about $0.13 \%$ to $0.2 \%$ of the total ADM mass, well below the $\sim 3 \%$ found in short inspirals of non-spinning equal-mass binaries in $D=4$ [72]. Also, the energy is radiated entirely in the form of a brief burst during merger with no significant analogue of the inspiral contribution clearly perceptible in four-dimensional binaries; see e.g. Fig. 18 in Ref. [91]. The relatively weaker gravitational attraction in $D=6$ implies slow orbital motion and, hence, inefficient generation of gravitational waves except for the final plunge phase. This is confirmed by the scattering configuration B5: without the plunge phase, the radiated energy drops by more than an order of magnitude.

\section{Conclusions}

In this paper we have (i) developed and tested a module for numerically computing apparent horizons for topologically spherical BHs in $D>4$ dimensions with $S O(D-3)$ isometry, (ii) generalised the construction of higher-dimensional Bowen-York type data given in [86] to orbiting $\mathrm{BH}$ binaries, and (iii) used these data and the horizon diagnostics to explore the dynamics of inspiraling $\mathrm{BH}$ binaries in higher dimensional general relativity. Our main findings are summarised as follows.

- The computation of $\mathrm{AHs}$ in $D=4$ dimensions based on the techniques of Alcubierre [44] generalise straightforwardly to higher-dimensional numerical relativity employing the modified cartoon method (Secs. 3.1, 3.2).

- Mass and spin can be computed directly from the AH's surface area and equatorial circumference (Sec. 3.3).

- For analytic single BH data, the AH finder obtains the correct values with an accuracy of a few times $10^{-5}$ even for modest grid resolutions (Tables 1, 2). In binary evolutions, the error budget is dominated by the discretization error of the numerical simulation rather than the $\mathrm{AH}$ finder itself, leading to uncertainties of a few percent in the cases studied here (Fig. 2, Table 3).

- The Bowen-York type initial data constructed for axisymmetric BH binaries in $D>4$ in Ref. [86] can be generalised straightforwardly to orbiting binaries (Appendix A).

- In contrast to the $D=4$ dimensional case, we find all BH binary systems modelled here in $D=6$ to either merge after completing about one orbit or less, or to scatter off each other without forming a common horizon. Without further finetuning of the initial parameters, we have not found binaries completing multiple orbits. This observation indicates that the absence of stable circular orbits around higherdimensional BHs generalises to binaries with non-linear dynamics (Fig. 1).

- The orbiting binaries here simulated in $D=6$ spacetime dimensions radiate about $0.13 \%$ to $0.2 \%$ of the ADM energy in gravitational waves, about twice as 
much as in head-on collisions but over an order of magnitude less than their $D=4$ counterparts. The energy is almost exclusively radiated in the brief plunge-merger phase with no analogue to the inspiral contribution present in $D=4$ (upper panel in Fig. 2, Table 3).

Our numerical study provides a first exploration into the behaviour of orbiting $\mathrm{BH}$ binaries in higher dimensions. Clearly, a much larger set of runs is required for a comprehensive understanding, especially of the behaviour near the scattering threshold and the dependency on the number of spacetime dimensions $D$. Nevertheless, our results already demonstrate the qualitatively different nature of orbiting $\mathrm{BH}$ binaries in higher dimensions. We opened this work with the observation that the number $D$ is merely a free parameter in the theory of general relativity. As far as the dynamics of binary BH spacetimes are concerned, however, the case $D=4$ appears to be as special as the computation of geodesics around BHs suggests.

Aside from studying these dynamics in more detail through larger sets of simulations, our work points to various other extensions. These include the modelling of spinning binaries, $\mathrm{BH}$ collisions with relatively small impact parameter but much larger initial boosts, the extraction of angular momentum radiated in gravitational waves and a multipolar analysis generalising the computation of Kodama-Ishibashi [92] modes from the axisymmetric case of Ref. [93] to the case of orbiting binaries.

\section{Acknowledgments}

We thank Pau Figueras for the Myers-Perry initial data in Kerr-Schild coordinates and Nathan Johnson-McDaniel and Markus Kunesch for very helpful discussions on the topic. We acknowledge financial support provided under the European Union's H2020 ERC Consolidator Grant "Matter and strong-field gravity: New frontiers in Einstein's theory" grant agreement no. MaGRaTh-646597 funding from the European Union's Horizon 2020 research and innovation programme under the Marie Skłodowska-Curie grant agreement No 690904, the COST Action Grant No. CA16104, from STFC Consolidator Grant No. ST/L000636/1, the SDSC Comet and TACC Stampede2 clusters through NSF-XSEDE Award Nos. PHY-090003, by PRACE for awarding us access to MareNostrum at Barcelona Supercomputing Center (BSC), Spain under Grant No.2016163948, and Cambridge's Cosmos Shared Memory system through BIS Grant No. ST/J005673/1 and STFC Grant Nos. ST/H008586/1, ST/K00333X/1. W.G.C. acknowledges support by a STFC studentship and D.W. support by a Trinity College Summer Research Fellowship.

\section{Appendix A. Numerically Constructed Initial Data for Non Head-On Collisions}

In section 5.1 we have used initial data similar to the well known Bowen-York initial data in $4 \mathrm{D}$ to initialise $\mathrm{BHs}$ with linear momentum both parallel to and transverse to the direction towards the other $\mathrm{BH}$. The form of this data for higher dimensions was initially proposed in [85, 94], and was explicitly given for momentum only parallel to the direction of the other $\mathrm{BH}$, and implemented in the reduction by isometry dimensional reduction scheme in [86]. Here we explicitly describe the implementation of this data in the modified cartoon formalism, with arbitrary initial momentum in the computational domain. 
In order to construct initial data, we must solve the $D$ constraint equations, one Hamiltonian constraint, and $D-1$ momentum constraints,

$$
\begin{aligned}
& \mathcal{H}=R+K^{2}-K^{I J} K_{I J}=0, \\
& \mathcal{M}^{I}=D_{J}\left(K^{I J}-\gamma^{I J} K\right)=0 .
\end{aligned}
$$

We first decompose the metric in the manner of York and Lichnerowicz, [95, 96, 97, 98]:

$$
\gamma_{I J}=\psi^{\frac{4}{D-3}} \hat{\gamma}_{I J}, \quad K_{I J}=\psi^{-2} \hat{A}_{I J}+\frac{1}{D-1} \gamma_{I J} K
$$

where $\psi$ is a conformal factor, and $K=\gamma^{I J} K_{I J}$ is the trace of the extrinsic curvature. We assume that the metric is conformally flat, $\hat{\gamma}_{I J}=\delta_{I J}$, and the maximal slicing condition, that is, $K=0$, which leads to a decoupling of the Hamiltonian and momentum constraints,

$$
\begin{aligned}
& \partial_{I} \hat{A}^{I J}=0, \\
& \hat{\triangle} \psi+\frac{D-3}{4(D-2)} \psi^{(-3 D-5) /(D-3)} \hat{A}^{I J} \hat{A}_{I J}=0 .
\end{aligned}
$$

Here $\hat{\triangle}$ is the flat space Laplacian $\hat{\triangle} \equiv \hat{\gamma}^{I J} \partial_{I} \partial_{J}$. Note that the indices on conformally rescaled quantities, such as $\hat{A}^{I J}$ are raised with the conformal metric $\hat{\gamma}^{I J}$. Following Yoshino et al. [85], we take an ansatz for $\hat{A}_{I J}$ giving a single boosted $\mathrm{BH}$,

$$
\begin{aligned}
\hat{A}^{I J}= & \frac{4 \pi(D-1)}{(D-2) \Omega_{D-2}} \frac{1}{r^{D-2}} \\
& {\left[n^{I} P^{J}+n^{J} P^{I}-n_{M} P^{M} \hat{\gamma}^{I J}+(D-3) n^{I} n^{J} P^{M} n_{M}\right], }
\end{aligned}
$$

where $P^{I}$ corresponds to the ADM momentum of the $\mathrm{BH}$ and $n^{I}$ is the normal radial vector in Cartesian coordinates given by

$$
n^{I} \equiv \frac{x^{I}-x_{0}^{I}}{r}, \quad r^{2}=\delta_{M N} x^{M} x^{N},
$$

and $x_{0}^{I}$ denotes the position of the $\mathrm{BH}$. This ansatz is chosen such that it solves Eq. (A.4), and reproduces the ADM momentum of a boosted BH. We are then left with the task of solving Eq. (A.5), an elliptic PDE for $\psi$. As in 4D for Bowen-York data, we decompose $\psi$ into a Brill-Lindquist component $\psi_{B L}$ [99], which on its own gives initial data for a static $\mathrm{BH}$, giving the spacetime approximately the correct ADM mass, and a correction $u$. For a single $\mathrm{BH}$, this is given by

$$
\psi=\psi_{B L}+u=1+\frac{\mu}{4 r^{D-3}}+u,
$$

where $\mu$ is the Schwarzschild-Tangherlini mass parameter, and $r$ is the radial distance from the BH. If we wish to solve for more than one BH, clearly Eq. (A.4) is linear in $\hat{A}^{I J}$, so we can superpose 2 sets of extrinsic curvature

$$
\hat{A}_{\text {tot }}^{I J}=\hat{A}_{(1)}^{I J}\left(P_{(1)}^{K}, n_{(1)}^{L}\right)+\hat{A}_{(2)}^{I J}\left(P_{(2)}^{K}, n_{(2)}^{L}\right),
$$

where $P_{(i)}^{K}$ and $n_{(i)}^{L}$ are the linear momentum, and radial vector respectively, corresponding to the $i$ th $\mathrm{BH}$. We then let $\psi$ take the same form as above, with the Brill-Lindquist term modified to include a contribution from the second $\mathrm{BH}$,

$$
\psi=1+\frac{\mu_{(1)}}{4 r_{(1)}^{D-3}}+\frac{\mu_{(2)}}{4 r_{(2)}^{D-3}}+u,
$$


where $\mu_{(i)}$ and $r_{(i)}$ are respectively the mass parameter of, and radial distance from, the $i$ th BH. Now we must solve Eq. (A.5) for $u$, which we achieve using a spectral elliptic PDE solver detailed in $[87,86]$, implemented in the CaCtus thorn TwoPunctures.

The final step is to identify how $\hat{A}^{I J}$ simplifies within the symmetry restrictions we place on our spacetime in implementing the modified cartoon formalism. Without loss of generality let us consider the case of a single $\mathrm{BH}$ with initial momentum and position

$$
P^{I}=\left(P_{x}, P_{y}, 0,0, \ldots, 0\right), \quad x_{0}^{I}=\left(x_{0}, 0,0,0, \ldots, 0\right) .
$$

Let us define for convenience

$$
\hat{a}^{I J}=n^{I} P^{J}+n^{J} P^{I}-n_{M} P^{M} \hat{\gamma}^{I J}+(D-3) n^{I} n^{J} P^{M} n_{M},
$$

so that

$$
\hat{A}^{I J}=\frac{4 \pi(D-1)}{(D-2) \Omega_{D-2} r^{D-2}} \hat{a}^{I J} .
$$

In the modified cartoon approach, we have $w_{4}=\ldots=w_{D-1}=0$, so that the radial vector has non-vanishing components only in the $x, y$ and $z$ directions. Furthermore, we use Cartesian coordinates, so that the expressions we insert into Eq. (A.12) are given by Eq. (A.11) as well as

$$
\begin{aligned}
& n^{I}=\left(\frac{x-x_{0}}{r}, \frac{y}{r}, \frac{z}{r}, 0, \ldots, 0\right), \\
& \hat{\gamma}_{I J}=\delta_{I J} .
\end{aligned}
$$

We can now calculate the individual terms in Eq. (A.12), firstly for terms inside the computational domain,

$$
\begin{aligned}
& n^{i} P^{j}=\left(\begin{array}{ccc}
P_{x} \frac{x-x_{0}}{r} & P_{y} \frac{x-x_{0}}{r} & 0 \\
P_{x} \frac{y}{r} & P_{y} \frac{y}{r} & 0 \\
P_{x} \frac{z}{r} & P_{y} \frac{z}{r} & 0
\end{array}\right), \quad n^{j} P^{i}=\left(\begin{array}{ccc}
P_{x} \frac{x-x_{0}}{r} & P_{x} \frac{y}{r} & P_{x} \frac{z}{r} \\
P_{y} \frac{x-x_{0}}{r} & P_{y} \frac{y}{r} & P_{y} \frac{z}{r} \\
0 & 0 & 0
\end{array}\right), \\
& n_{M} P^{M} \hat{\gamma}^{i j}=\left(\begin{array}{ccc}
P_{x} \frac{x-x_{0}}{r}+P_{y} \frac{y}{r} & 0 & 0 \\
0 & P_{x} \frac{x-x_{0}}{r}+P_{y} \frac{y}{r} & 0 \\
0 & 0 & P_{x} \frac{x-x_{0}}{r}+P_{y} \frac{y}{r}
\end{array}\right), \\
& (D-3) n^{i} n^{j} n_{M} P^{M}=(D-3)\left(P_{x} \frac{x-x_{0}}{r}+P_{y} \frac{y}{r}\right)\left(\begin{array}{ccc}
\frac{\left(x-x_{0}\right)^{2}}{r^{2}} & \frac{\left(x-x_{0}\right) y}{r^{2}} & \frac{\left(x-x_{0}\right) z}{r^{2}} \\
\frac{\left(x-x_{0}\right) y}{r^{2}} & \frac{y^{2}}{r^{2}} & \frac{y z}{r^{2}} \\
\frac{\left(x-x_{0}\right) z}{r^{2}} & \frac{y z}{r^{2}} & \frac{z^{2}}{r^{2}}
\end{array}\right) .
\end{aligned}
$$


We thus obtain the components

$$
\begin{aligned}
\hat{a}^{22} & =P_{y} \frac{y}{r^{3}}\left((D-2) y^{2}+z^{2}+\left(x-x_{0}\right)^{2}\right)+P_{x} \frac{x-x_{0}}{r^{3}}\left((D-4) y^{2}-z^{2}-\left(x-x_{0}\right)^{2}\right), \\
\hat{a}^{23} & =P_{y} \frac{z}{r^{3}}\left((D-2) y^{2}+z^{2}+\left(x-x_{0}\right)^{2}\right)+(D-3) P_{x} \frac{y z}{r^{3}}\left(x-x_{0}\right), \\
\hat{a}^{12} & =P_{y} \frac{x-x_{0}}{r^{3}}\left((D-2) y^{2}+z^{2}+\left(x-x_{0}\right)^{2}\right)+P_{x} \frac{y}{r^{3}}\left(y^{2}+z^{2}+(D-2)\left(x-x_{0}\right)^{2}\right), \\
\hat{a}^{33} & =\left(P_{y} \frac{y}{r^{3}}+P_{x} \frac{x-x_{0}}{r^{3}}\right)\left(-y^{2}+(D-4) z^{2}-\left(x-x_{0}\right)^{2}\right), \\
\hat{a}^{13} & =(D-3) P_{y} \frac{y z}{r^{3}}\left(x-x_{0}\right)+P_{x} \frac{z}{r^{3}}\left(y^{2}+z^{2}+(D-2)\left(x-x_{0}\right)^{2}\right), \\
\hat{a}^{11} & =P_{y} \frac{y}{r^{3}}\left(-y^{2}-z^{2}+(D-4)\left(x-x_{0}\right)^{2}\right)+P_{x} \frac{x-x_{0}}{r^{3}}\left(y^{2}+z^{2}+(D-2)\left(x-x_{0}\right)^{2}\right) .
\end{aligned}
$$

Finally we calculate the off-domain components,

$$
\hat{a}^{a b}=\left(-P_{x} \frac{x-x_{0}}{r}-P_{y} \frac{y}{r}\right) \delta_{a b} \quad \Rightarrow \quad \hat{a}^{w w}=-P_{x} \frac{x-x_{0}}{r}-P_{y} \frac{y}{r},
$$

and we note that $\hat{a}^{I J}$ is tracefree as expected.

\section{References}

[1] Clifford M. Will. The Confrontation between General Relativity and Experiment. Living Rev. Rel., 17:4, 2014.

[2] Emanuele Berti et al. Testing General Relativity with Present and Future Astrophysical Observations. Class. Quant. Grav., 32:243001, 2015. arXiv:1501.07274 [gr-qc].

[3] B. P Abbott et al. Observation of Gravitational Waves from a Binary Black Hole Merger. Phys. Rev. Lett., 116(6):061102, 2016. arXiv:1602.03837 [gr-qc].

[4] B. P. Abbott et al. Tests of general relativity with GW150914. Phys. Rev. Lett., 116(22):221101, 2016. arXiv:1602.03841 [gr-qc].

[5] B. P. Abbott et al. GW170817: Observation of Gravitational Waves from a Binary Neutron Star Inspiral. Phys. Rev. Lett., 119(16):161101, 2017.

[6] R. Emparan, R. Suzuki, and K. Tanabe. The large D limit of General Relativity. JHEP, 06:009, 2013. arXiv:1302.6382 [hep-th].

[7] T. Kaluza. Zum Unitätsproblem der Physik. Sitzungsber. Preuss. Akad. Wiss. Berlin (Math. Phys.), 1921:966-972, 1921.

[8] O. Klein. Quantum Theory and Five-Dimensional Theory of Relativity. (In German and English). Z. Phys., 37:895-906, 1926. [,76(1926)].

[9] M. B. Green, J. H. Schwarz, and E. Witten. Superstring Theory. Vol. 1: Introduction. Cambridge Monographs on Mathematical Physics. Cambridge University Press, Cambridge, UK, 1988.

[10] B. R. Greene, D. R. Morrison, and J. Polchinski. String theory. Proc. Nat. Acad. Sci., 95:1103911040, 1998.

[11] C. Rovelli. Loop quantum gravity. Living Rev. Relativity, 11:5, 2008.

[12] J. M. Maldacena. The large N limit of superconformal field theories and supergravity. Adv. Theor. Math. Phys., 2:231, 1997. hep-th/9711200.

[13] E. Witten. Anti-de Sitter space, thermal phase transition, and confinement in gauge theories. Adv. Theor. Math. Phys., 2:505-532, 1998. hep-th/9803131.

[14] P. M. Chesler and L. G. Yaffe. Horizon formation and far-from-equilibrium isotropization in supersymmetric Yang-Mills plasma. Phys. Rev. Lett., 102:211601, 2009. arXiv:0812.2053 [hep-th].

[15] S. A. Hartnoll. Lectures on holographic methods for condensed matter physics. Class. Quant. Grav., 26:224002, 2009. arXiv:0903.3246 [hep-th].

[16] Ignatios Antoniadis. A Possible new dimension at a few TeV. Phys. Lett. B, 246:377-384, 1990.

[17] N. Arkani-Hamed, S. Dimopoulos, and G. R. Dvali. The hierarchy problem and new dimensions at a millimeter. Phys. Lett. B, 429:263-272, 1998. hep-ph/9803315. 
[18] I. Antoniadis, N. Arkani-Hamed, S. Dimopoulos, and G. R. Dvali. New dimensions at a millimeter to a Fermi and superstrings at a TeV. Phys. Lett. B, 436:257-263, 1998. hep$\mathrm{ph} / 9804398$.

[19] L. Randall and R. Sundrum. A large mass hierarchy from a small extra dimension. Phys. Rev. Lett., 83:3370-3373, 1999. hep-ph/9905221.

[20] L. Randall and R. Sundrum. An alternative to compactification. Phys. Rev. Lett., 83:4690-4693, 1999. hep-th/9906064.

[21] Morad Aaboud et al. Search for new phenomena in dijet events using $37 \mathrm{fb}^{-1}$ of $p p$ collision data collected at $\sqrt{s}=13 \mathrm{TeV}$ with the ATLAS detector. Phys. Rev., D96(5):052004, 2017.

[22] A. M. Sirunyan et al. Search for black holes and sphalerons in high-multiplicity final states in proton-proton collisions at $\sqrt{s}=13 \mathrm{TeV} .2018$.

[23] T. Banks and W. Fischler. A Model for High Energy Scattering in Quantum Gravity. 1999. hep-th/9906038.

[24] S. B. Giddings and S. Thomas. High energy colliders as black hole factories: The end of short distance physics. Phys. Rev. D, 65:056010, 2002. hep-ph/0106219.

[25] S. Dimopoulos and G. Landsberg. Black Holes at the LHC. Phys. Rev. Lett., 87:161602, 2001. hep-th/0106295.

[26] V. Cardoso, L. Gualtieri, C. Herdeiro, and U. Sperhake. Exploring New Physics Frontiers Through Numerical Relativity. Living Rev. Relativity, 18:1, 2015. arXiv:1409.0014 [gr-qc].

[27] R. Emparan and H. S. Reall. A Rotating black ring solution in five-dimensions. Phys. Rev. Lett., 88:101101, 2002. hep-th/0110260.

[28] H. Elvang and P. Figueras. Black Saturn. JHEP, 0705:050, 2007. hep-th/0701035.

[29] R. Emparan and R. C. Myers. Instability of ultra-spinning black holes. JHEP, 0309:025, 2003. hep-th/0308056.

[30] L. Lehner and F. Pretorius. Black Strings, Low Viscosity Fluids, and Violation of Cosmic Censorship. Phys. Rev. Lett., 105:101102, 2010. arXiv:1006.5960 [hep-th].

[31] M. Shibata and H. Yoshino. Bar-mode instability of rapidly spinning black hole in higher dimensions: Numerical simulation in general relativity. Phys. Rev. D, 81:104035, 2010. arXiv:1004.4970 [gr-qc].

[32] P. Figueras, M. Kunesch, and S. Tunyasuvunakool. End Point of Black Ring Instabilities and the Weak Cosmic Censorship Conjecture. Phys. Rev. Lett., 116(7):071102, 2016. arXiv:1512.04532 [hep-th].

[33] P. Figueras, M. Kunesch, L. Lehner, and S. Tunyasuvunakool. End Point of the Ultraspinning Instability and Violation of Cosmic Censorship. Phys. Rev. Lett., 118(15):151103, 2017.

[34] Roberto Emparan and Kentaro Tanabe. Universal quasinormal modes of large D black holes. Phys. Rev., D89(6):064028, 2014.

[35] R. Emparan, T. Shiromizu, R. Suzuki, K. Tanabe, and T. Tanaka. Effective theory of Black Holes in the 1/D expansion. JHEP, 06:159, 2015. arXiv:1504.06489 [hep-th].

[36] Roberto Emparan, Ryotaku Suzuki, and Kentaro Tanabe. Evolution and End Point of the Black String Instability: Large D Solution. Phys. Rev. Lett., 115(9):091102, 2015.

[37] R. Emparan and H. S. Reall. Black Holes in Higher Dimensions. Living Reviews in Relativity, 11(6), 2008. http://www.livingreviews.org/lrr-2008-6.

[38] Frans Pretorius. Binary Black Hole Coalescence. In M. Colpi et al., editor, Physics of Relativistic Objects in Compact Binaries: From Birth to Coalescence. Springer, New York, 2009. arXiv:0710.1338 [gr-qc].

[39] H. Yoshino and M. Shibata. Exploring Higher-Dimensional Black Holes in Numerical Relativity. Prog.Theor.Phys.Suppl., 190:282-303, 2011.

[40] Ulrich Sperhake. Numerical relativity in higher dimensions. Int. J. Mod. Phys. D, 22:1330005, 2013. arXiv:1301.3772 [gr-qc].

[41] L. Barack et al. Black holes, gravitational waves and fundamental physics: a roadmap. 2018.

[42] Peter Diener. A New general purpose event horizon finder for 3-D numerical space-times. Class. Quant. Grav., 20:4901-4918, 2003. gr-qc/0305039.

[43] M. I. Cohen, H. P. Pfeiffer, and M. A. Scheel. Revisiting Event Horizon Finders. Class. Quant. Grav., 26:035005, 2009. arXiv:0809.2628 [gr-qc].

[44] M. Alcubierre, S. Brandt, Bernd Brügmann, C. Gundlach, J. Masso, E. Seidel, and P. Walker. Test beds and applications for apparent horizon finders in numerical relativity. Class. Quant. Grav., 17:2159-2190, 2000. gr-qc/9809004.

[45] C. Gundlach. Pseudospectral apparent horizon finders: An Efficient new algorithm. Phys. Rev. $D, 57: 863-875,1998$. gr-qc/9707050.

[46] E. Schnetter. Finding apparent horizons and other two surfaces of constant expansion. Class. Quant. Grav., 20:4719-4737, 2003. gr-qc/0306006. 
[47] J. Thornburg. Finding apparent horizons in numerical relativity. Phys. Rev. D, 54:4899-4918, 1996. gr-qc/9508014.

[48] J. Thornburg. A Fast apparent horizon finder for three-dimensional Cartesian grids in numerical relativity. Class. Quant. Grav., 21:743-766, 2004. gr-qc/0306056.

[49] J. Thornburg. Event and Apparent Horizon Finders in 3+1 Numerical Relativity. Living Reviews in Relativity, 10(3), 2007. gr-qc/0512169.

[50] H. Okawa, K.-i. Nakao, and M. Shibata. Is super-Planckian physics visible? Scattering of black holes in 5 dimensions. Phys. Rev. D, 83:121501, 2011. arXiv:1105.3331 [gr-qc].

[51] S. Tunyasuvunakool. Applications of Numerical Relativity Beyond Astrophysics. PhD thesis, University of Cambridge, 2017.

[52] R. Arnowitt, S. Deser, and C. W. Misner. The dynamics of general relativity. In L. Witten, editor, Gravitation an introduction to current research, pages 227-265. John Wiley, New York, 1962. gr-qc/0405109.

[53] Jr. J. W. York. Kinematics and dynamics of general relativity. In L. Smarr, editor, Sources of Gravitational Radiation, pages 83-126. Cambridge University Press, Cambridge, 1979.

[54] E. Gourgoulhon. 3+1 Formalism and Bases of Numerical Relativity. 2007. gr-qc/0703035.

[55] F. Pretorius. Numerical relativity using a generalized harmonic decomposition. Class. Quantum Grav., 22:425-452, 2005. gr-qc/0407110.

[56] H. Yoshino and M. Shibata. Higher-Dimensional Numerical Relativity: Current Status. Prog.Theor.Phys.Suppl., 189:269-310, 2011.

[57] T. W. Baumgarte and S. L. Shapiro. On the Numerical integration of Einstein's field equations. Phys. Rev. D, 59:024007, 1998. gr-qc/9810065.

[58] M. Shibata and T. Nakamura. Evolution of three-dimensional gravitational waves: Harmonic slicing case. Phys. Rev. D, 52:5428-5444, 1995.

[59] William G. Cook, Pau Figueras, Markus Kunesch, Ulrich Sperhake, and Saran Tunyasuvunakool. Dimensional reduction in numerical relativity: Modified cartoon formalism and regularization. In 3rd Amazonian Symposium on Physics and 5th NRHEP Network Meeting: Celebrating 100 Years of General Relativity Belem, Brazil, September 28-October 2, 2015, volume 25, page 1641013, 2016. arXiv:1603.00362 [gr-qc].

[60] Allen, G. and Goodale, T. and Massó, J. and Seidel, E. The Cactus Computational Toolkit and Using Distributed Computing to Collide Neutron Stars. In Proceedings of Eighth IEEE International Symposium on High Performance Distributed Computing, HPDC-8, Redondo Beach, 1999, , 1999. IEEE Press.

[61] Cactus Computational Toolkit homepage: http://www.cactuscode.org/.

[62] T. W. Baumgarte, G. B. Cook, M. A. Scheel, S. L. Shapiro, and S. A. Teukolsky. Implementing an apparent-horizon finder in three dimensions. Phys. Rev. D, 54:4849, 1996. gr-qc/9606010.

[63] W. H. Press, B. P. Flannery, S. A. Teukolsky, and W. T. Vetterling. Numerical Recipes. Cambridge University Press, Cambridge, 1989.

[64] S. W. Hawking and G. F. R. Ellis. The Large Scale Structure of Space-Time. Cambridge University Press, 1973.

[65] Gregory J. Galloway. Constraints on the topology of higher dimensional black holes. 2011.

[66] F.R. Tangherlini. Schwarzschild field in $\mathrm{n}$ dimensions and the dimensionality of space problem. Nuovo Cim., 27:636-651, 1963.

[67] R. C. Myers and M. J. Perry. Black Holes in Higher Dimensional Space-Times. Annals Phys., 172:304, 1986.

[68] U. Sperhake. Binary black-hole evolutions of excision and puncture data. Phys. Rev. D, 76:104015, 2007. gr-qc/0606079.

[69] U. Sperhake, E. Berti, V. Cardoso, J. A. González, B. Brügmann, and M. Ansorg. Eccentric binary black-hole mergers: The transition from inspiral to plunge in general relativity. Phys. Rev. D, 78:064069, 2008. arXiv:0710.3823 [gr-qc].

[70] E. Schnetter, S. H. Hawley, and I. Hawke. Evolutions in 3-D numerical relativity using fixed mesh refinement. Class. Quant. Grav., 21:1465-1488, 2004. gr-qc/0310042.

[71] Carpet Code homepage: http://www. carpetcode.org/.

[72] J. G. Baker, J. Centrella, D.-I. Choi, M. Koppitz, and J. van Meter. Gravitational-Wave Extraction from an inspiraling Configuration of Merging Black Holes. Phys. Rev. Lett., 96:111102, 2006. gr-qc/0511103.

[73] M. Campanelli, C. O. Lousto, P. Marronetti, and Y. Zlochower. Accurate Evolutions of Orbiting Black-Hole Binaries without Excision. Phys. Rev. Lett., 96:111101, 2006. gr-qc/0511048.

[74] M. Zilhão, H. Witek, U. Sperhake, V. Cardoso, L. Gualtieri, C. Herdeiro, and A. Nerozzi. Numerical relativity for D dimensional axially symmetric space-times: formalism and code tests. Phys. Rev. D, 81:084052, 2010. arXiv:1001.2302 [gr-qc]. 
[75] William G. Cook and Ulrich Sperhake. Extraction of gravitational-wave energy in higher dimensional numerical relativity using the Weyl tensor. Class. Quant. Grav., 34(3):035010, 2017. arXiv:1609.01292 [gr-qc].

[76] K. A. Dennison, J. P. Wendell, T. W. Baumgarte, and J. D. Brown. Trumpet slices of the Schwarzschild-Tangherlini spacetime. Phys. Rev. D, 82:124057, 2010. arXiv:1010.5723 [grqc].

[77] M. Shibata, H. Okawa, and T. Yamamoto. High-velocity collisions of two black holes. Phys. Rev. D, 78:101501(R), 2008. arXiv:0810.4735 [gr-qc].

[78] U. Sperhake, V. Cardoso, F. Pretorius, E. Berti, T. Hinderer, and N. Yunes. Cross section, final spin and zoom-whirl behavior in high-energy black hole collisions. Phys. Rev. Lett., 103:131102, 2009. arXiv:0907.1252 [gr-qc].

[79] V. Cardoso, A. S. Miranda, E. Berti, H. Witek, and V. T. Zanchin. Geodesic stability, Lyapunov exponents and quasinormal modes. Phys. Rev. D, 79:064016, 2009. arXiv:0812.1806 [hep-th].

[80] F. Pretorius and D. Khurana. Black Hole Mergers and Unstable Circular Orbits. Class. Quantum Grav., 24:S83-S108, 2007. gr-qc/0702084.

[81] J. Healy, J. Levin, and D. Shoemaker. Zoom-Whirl Orbits in Black Hole Binaries. Phys. Rev. Lett., 103:131101, 2009. arXiv:0907.0671 [gr-qc].

[82] R. Gold and B. Brügmann. Radiation from low-momentum zoom-whirl orbits. Class. Quant. Grav., 27:084035, 2010. arXiv:0911.3862 [gr-qc].

[83] J. M. Bowen and Jr. J. W. York. Time-asymmetric initial data for black holes and black-hole collisions. Phys. Rev. D, 21:2047-2056, 1980.

[84] G. B. Cook. Initial Data for Numerical Relativity. Living Reviews in Relativity, 3(5), 2000. gr-qc/0007085.

[85] H. Yoshino, T. Shiromizu, and M. Shibata. Close-slow analysis for head-on collision of two black holes in higher dimensions: Bowen-York initial data. Phys. Rev. D, 74:124022, 2006. gr-qc/0610110.

[86] M. Zilhão, M. Ansorg, V. Cardoso, L. Gualtieri, C. Herdeiro, U. Sperhake, and H. Witek. Higher-dimensional puncture initial data. Phys. Rev. D, 84:084039, 2011. arXiv:1109.2149 [gr-qc].

[87] M. Ansorg, B. Brügmann, and W. Tichy. A single-domain spectral method for black hole puncture data. Phys. Rev. D, 70:064011, 2004. gr-qc/0404056.

[88] U. Sperhake, B. Brügmann, D. Müller, and C. F. Sopuerta. 11-orbit inspiral of a mass ratio 4:1 black-hole binary. Class. Quant. Grav., 28:134004, 2011. arXiv:1012.3173 [gr-qc].

[89] H. Witek, H. Okawa, V. Cardoso, L. Gualtieri, C. Herdeiro, M. Shibata, U. Sperhake, and M. Zilhão. Higher dimensional Numerical Relativity: code comparison. Phys. Rev. D, 90(8):084014, 2014. arXiv:1406.2703 [gr-qc].

[90] P. C. Peters and J. Mathews. Gravitational Radiation from Point Masses in a Keplerian Orbit. Phys. Rev., 131:435-439, 1963.

[91] A. Buonanno, G. B. Cook, and F. Pretorius. Inspiral, merger and ring-down of equal-mass black-hole binaries. Phys. Rev. D, 75:124018, 2007. gr-qc/0610122.

[92] H. Kodama and A. Ishibashi. A master equation for gravitational perturbations of maximally symmetric black holes in higher dimensions. Prog. Theor. Phys., 110:701-722, 2003. hepth/0305147.

[93] H. Witek, M. Zilhão, L. Gualtieri, V. Cardoso, C. Herdeiro, A. Nerozzi, and U. Sperhake. Numerical relativity for D dimensional space-times: head-on collisions of black holes and gravitational wave extraction. Phys. Rev. D, 82:104014, 2010. arXiv:1006.3081 [gr-qc].

[94] H. Yoshino, T. Shiromizu, and M. Shibata. The close limit analysis for head-on collision of two black holes in higher dimensions: Brill-Lindquist initial data. Phys. Rev. D, 72:084020, 2005. gr-qc/0508063.

[95] A. Lichnerowicz. L'integration des équations de la gravitation relativiste et le problème des $n$ corps. J. Math. Pures et Appl., 23:37-63, 1944.

[96] Jr. J. W. York. Gravitational degrees of freedom and the initial-value problem. Phys. Rev. Lett., 26:1656-1658, 1971.

[97] Jr. J. W. York. Role of conformal three-geometry in the dynamics of gravitation. Phys. Rev. Lett., 28:1082-1085, 1972.

[98] Jr. J. W. York. Conformally invariant orthogonal decomposition of symmetric tensors on Riemannian manifolds and the initial-value problem of general relativity. J. Math. Phys., 14:456-464, 1973.

[99] D. R. Brill and R. W. Lindquist. Interaction Energy in Geometrostatics. Phys. Rev., 131:471$476,1963$. 